\title{
Burnout en trabajadores de hospitales comunitarios o de baja complejidad: una comparacion de género y de nivel de escolaridad $^{1}$
}

Burnout in workers from community or low complex
hospitals gender and school leve comparisson

Maria Margarita Chiang ${ }^{2}$

Marcelo Sigoña ${ }^{3}$

Resumen

Este estudio analiza el nivel de burnout en los trabajadores de hospitales comunitarios o de baja complejidad. La población sobre la cual se aplicó el estudio fue 400 individuos, con una tasa de respuesta de $42 \%$. Se utilizó el Cuestionario para la evaluación del síndrome de quemarse por el trabajo (CESQT). La fiabilidad de las escalas en todos los casos es adecuada. Los resultados muestran que un 76\% de los funcionarios no presenta burnout, un $18 \%$ presenta trastorno pero no está inhabilitado clínicamente y un $6 \%$ está inhabilitado para trabajar sin tratamiento clínico. El nivel de escolaridad influye en la aparición del burnout, se podría esperar que a niveles de escolaridad universitaria se tuviera mayores recursos para enfrentar el burnout.

Palabras clave: Burnout, hospital comunitario, género y nivel de escolaridad.

1 Proyecto de investigación 072116-4/R Universidad del Bío-Bío, Concepción, Chile.

2 Doctora en Recursos Humanos. Universidad del Bío-Bío. Concepción. Chile. E-mail: mchiang@ ubiobio.cl

3 Licenciado en Psicología. Servicio Salud Nuuble. Chillán. Chile. E-mail: rolando.sigona@redsalud.gov.cl. 


\begin{abstract}
This paper presents the analysis of the level of burnout in workers the community hospitals of low complexity. The population on which applied the study was 400 individuals, with a response rate of $42 \%$. The instrument used for was questionnaire for the assessment of burnout syndrome (CESQT). The reliability of the scales in all cases is adequate. The results show that $76 \%$ of staff do not have burnout, $18 \%$ with disorder but is not clinically disabled and $6 \%$ are unable to work without clinical treatment. The level of education influences the occurrence of burnout, we might expect a university education levels increased resources to deal with burnout.
\end{abstract}

Key words: Burnout, community hospital, gender and level education. 
Planteamiento del problema y perspectiva teórica

El problema a estudiar es el nivel de burnout en los trabajadores de hospitales comunitarios o de baja complejidad ubicados en la zona rural de la provincia de Nuble. Es importante estudiar la salud mental de los funcionarios de los hospitales porque incide en la calidad del servicio prestado a los pacientes, así, primero es necesario conocer cuál es la situación de la salud de los trabajadores y luego gestionar cómo ayudarlos a superar los problemas que se detecten.

El burnout o síndrome de estar quemado por el trabajo (SQT) constituye uno de los daños laborales de carácter psicosocial más importantes en la sociedad actual. El elevado ritmo de vida, la transformación de los mercados y la estructura del sector económico -caracterizados por un incremento del trabajo emocional y mental-, las exigencias de mayor calidad del trabajo, junto con la ruptura del contrato psicológico y los costos que el burnout supone para las personas y las organizaciones, ha despertado el interés en conocer y tomar medidas para prevenir este síndrome.

El estudio del burnout a nivel científico ha permitido el desarrollo del concepto hasta nuestros días. Originalmente, Freudenberger (1974) y Maslach (1976) consideraron el burnout como un síndrome que aparecía en individuos que trabajaban con personas o profesionales de servicios. Estaba compuesto por tres dimensiones básicas: agotamiento emocional; despersonalización; y reducida realización personal. Así, el síndrome de estar quemado por el trabajo puede entenderse como una respuesta prolongada a estresores crónicos a nivel personal y relacional en el trabajo, determinado a partir de las dimensiones conocidas como agotamiento, despersonalización y cinismo e ineficacia profesional (Maslach, Schaufeli \& Leiter, 2001).

Basándose en premisas de generalización y extensión del síndrome, Schaufeli y Enzmann (1998) ofrecen una definición sintética del burnout: estado mental, persistente, negativo y relacionado con el trabajo, en individuos "normales", que se caracteriza principalmente por agotamiento, 
que se acompaña de malestar, un sentimiento de reducida competencia y motivación, y el desarrollo de actitudes disfuncionales en el trabajo.

El burnout laboral es un síndrome psicológico que implica una respuesta prolongada a estresores interpersonales crónicos en el trabajo (Maslach, 2009). Una definición clásica de burnout indica que es un síndrome psicológico de agotamiento emocional, despersonalización y reducida realización personal que puede ocurrir en individuos normales que trabajan con personas. El agotamiento emocional se refiere a los sentimientos de no poder dar más de sí a nivel emocional y a una disminución de los propios recursos emocionales. La despersonalización se refiere a una respuesta de distancia negativa, sentimientos y conductas cínicas respecto a otras personas, que son normalmente los usuarios del servicio o del cuidado. La reducida realización personal se refiere a la disminución en los propios sentimientos de competenciay logro en el trabajo (Maslach, 1993). Esta situación se traduce en una serie de consecuencias tanto a nivel individual (agotamiento, fatiga crónica, cansancio, distancia mental, ansiedad, depresión, quejas psicosomáticas, incremento del uso de substancias tóxicas, generalización o "desbordamiento" a la vida privada, dudas respecto a la propia capacidad para desempeñar el trabajo), a nivel de trabajo (insatisfacción laboral, falta de compromiso organizacional e intención de abandonar la organización) como a nivel organizacional (incremento de la tasa de ausentismo y del abandono del trabajo, disminución del desempeño del trabajo y falta de calidad del servicio) (Schaufeli \& Buunk, 2002).

Recientemente, el estudio del burnout se ha ampliado a todo tipo de profesiones y grupos ocupacionales como trabajadores que laboran con datos (ej., teletrabajadores) o trabajadores que laboran con diferentes equipamientos (ej., operarios de producción de una industria) (Salanova, Schaufeli, Llorens, Peiró \& Grau, 2000). Además, el burnout también puede aparecer en muestras pre-ocupacionales: estudiantes universitarios, hablaríamos del síndrome de quemarse por los estudios que podría ocasionar depresión, malestar psicosocial e incluso el abandono de los estudios (Salanova, Martínez, Bresó, Llorens \& Grau, 2005). 
Se mantiene el carácter tridimensional del síndrome, pero con referencia a cualquier trabajo y son: agotamiento (fatiga producida por los excesivos esfuerzos psicológicos que se realizan en el trabajo sin tener en cuenta si la causa son las relaciones con las personas o con el trabajo más en general); cinismo (indiferencia y actitudes distantes respecto a los objetivos o utilidad del trabajo) y falta de eficacia profesional (tendencia a evaluar el propio trabajo de forma negativa que implicaría una reducción de las creencias de eficacia y habilidad para realizar su trabajo). Aunque generalmente altos niveles de agotamiento y cinismo o despersonalización y bajos niveles de eficacia profesional se han considerado indicadores de burnout, existe evidencia empírica que señala que el agotamiento y el cinismo constituyen el corazón o las dimensiones clave del burnout (Green, Walkey \& Taylor, 1991). Mientras que se ha mostrado el rol independiente de la eficacia profesional, considerándola como una dimensión más cercana a una variable de personalidad (más estable), como una forma de afrontamiento, o como un antecedente del burnout (Salanova, 2006).

Sin embargo, estudios más recientes realizados en muestras ocupacionales y pre-ocupacionales revelan que el burnout constituye un síndrome caracterizado por: agotamiento (relacionado con crisis en las relaciones entre la persona y el trabajo en general), distancia mental (que incluye tanto el cinismo -actitudes distantes hacia el trabajo en general-, como la despersonalización -actitudes distantes hacia las personas para y con las que se trabaja-), y la ineficacia profesional (sensación de no hacer adecuadamente las tareas y ser incompetente en el trabajo) (Salanova, 2006). Respecto a la medida de eficacia profesional, estudios recientes vienen a señalar la necesidad de medir "ineficacia" en lugar de "eficacia" utilizando ítems invertidos (Bresó, Salanova \& Schaufeli, 2007a; Schaufeli \& Salanova, 2007).

Junto con las tres variables, se observa en algunos individuos la aparición de sentimientos de culpa. La culpa es una emoción social vinculada a las relaciones interpersonales, relaciones íntimas o de proximidad en las que el individuo responde a las necesidades de la otra persona y no a un intercambio en el que se espera la reciprocidad (Baumeister, Stillwell \& Heatherton, 1994). La culpa tiene la función simbólica de reafirmar el 
compromiso con la otra persona y la responsabilidad de cuidarla, se utiliza como estrategia para influir sobre otros, y permite aliviar el estrés generado por la falta de equilibrio en los estados emocionales que resultan de los intercambios sociales. La culpa tiene efectos pro-sociales, pues motiva a las personas a desagraviar a los demás, a corregir sus errores y a disculparse.

La culpa aparece identificada como un síntoma característico del SQT en diversos estudios publicados en los primeros años de la década de los ochenta (Farber \& Miller 1981; Freunderberger, 1974; Maslach, 1982; Pines, 1983; Price \& Murphy, 1984). Maslach (1982) señala que es frecuente que los profesionales interpreten sus respuestas al estrés laboral crónico como una consecuencia de variables de personalidad. La aparición de ideas como "soy una mala persona", "soy incapaz para este trabajo", o "soy un incompetente" son comunes entre los profesionales que desarrollan el SQT, y es la causa que lleva a muchos a buscar algún tipo de terapia.

Gil-Monte (2005) establece una hipótesis acerca de la existencia de dos perfiles de individuos en el desarrollo del SQT. Por una parte, encontraremos individuos (Tipo 1) a los que las estrategias de afrontamiento cognitivas que permiten justificar la utilización de conductas proscritas por la ética (p. ej. justificación moral, difusión de la responsabilidad, atribución de culpa, deshumanización, etc.) les resultan de utilidad. Estos individuos no sentirán culpa con frecuencia por tratar a los clientes de manera desconsiderada o agresiva, o por no ajustarse a las expectativas del rol. Son profesionales que pueden mantenerse durante años en la organización sin desarrollar problemas individuales relevantes vinculados al estrés laboral, aunque con sus actitudes y conductas de indiferencia, apatía, irresponsabilidad, cinismo, indolencia, etc., deterioran la calidad del servicio de la organización y dan lugar a quejas por parte de los clientes sobre el trato recibido.

Por el contrario, el segundo perfil (Tipo 2) son individuos que experimentan remordimientos por no cumplir de manera adecuada las prescripciones del rol, por sentirse desgastados y no poder dar más de sí mismos, y por la utilización de estrategias de afrontamiento que conllevan un trato negativo e impersonal con los clientes. Estas estrategias no les 
resultan eficaces debido a la influencia de variables psicosociales (p. ej. orientación hacia otros, altruismo), o porque sienten que están violando algún tipo de código ético, o alguna norma derivada de las prescripciones del rol. En estos casos, los sentimientos de culpa intervienen en la aparición de las consecuencias del SQT. Probablemente, esos individuos experimentarán con frecuencia sentimientos de culpa hasta el punto de necesitar la desvinculación, acudirán con más frecuencia al médico, y manifestarán con mayor frecuencia e intensidad problemas psicosomáticos vinculados al estrés laboral. Incluso pueden desarrollar patologías del tipo crisis de ansiedad y depresión como consecuencias del SQT. Son personas que van a necesitar la ayuda de profesionales calificados para superar esas crisis que las incapacitan para el ejercicio de su trabajo.

La aparición de los sentimientos de culpa puede originar un círculo vicioso en el proceso de desarrollo del SQT que intensifica sus síntomas y lo hace más perverso. Los sentimientos de culpa llevarán a los profesionales a una mayor implicación laboral para disminuir sus remordimientos y su culpabilidad (Baumeister et al., 1994), pero como las condiciones del entorno laboral no cambian, se incrementará el deterioro cognitivo y emocional, y aparecerán de nuevo actitudes de indiferencia, indolencia y despersonalización. Este proceso desarrollará de nuevo sentimientos de culpa, o intensificará los existentes, manteniendo e incrementando la intensidad del SQT.

Este proceso contribuye a explicar la distinción establecida por Paine (1982) entre Burnout Stress Syndrome (Perfil 1) y Burnout Mental Disability (Perfil 2). El primero alude a un conjunto de sentimientos y conductas vinculados al estrés laboral que originan una forma moderada de malestar, pero que no incapacita al individuo para el ejercicio de su trabajo, aunque podría realizarlo mejor. El segundo, por el contrario, constituye con frecuencia un problema serio, con un cuadro clínico caracterizado por la existencia de síntomas de estrés y bajo rendimiento laboral, que es la fase final del proceso del SQT.

A modo de resumen, podemos decir que existen dos procesos que explican el proceso de desarrollo o etiología del burnout. El primero hace referencia al proceso de burnout desarrollado en profesionales de ayuda. 
En este caso, el burnout puede desencadenarse debido a las demandas interpersonales que implican el trato con pacientes/clientes/usuarios que incluso pueden llegar a consumirles emocionalmente. Para evitar el contacto con la fuente originaria del malestar se adopta la despersonalización como estrategia de afrontamiento, que llevaría en última instancia a una reducida realización personal.

El segundo proceso de desarrollo del burnout es de naturaleza más general, aplicable a cualquier ocupación. Se asume que, independientemente de la ocupación, una falta de confianza en las propias competencias resulta un factor crítico en el desarrollo del burnout (Cherniss, 1993). Investigaciones recientes han demostrado que, independientemente de la ocupación, el burnout se produce como consecuencia de crisis sucesivas de eficacia (ej., Llorens, García, \& Salanova, 2005). La presencia de altas demandas y pobres recursos podría generar estas crisis sucesivas de autoeficacia que con el tiempo producirían burnout; esto es, agotamiento, distancia mental (cinismo y despersonalización) e ineficacia profesional.

\section{Metodología}

El ámbito de estudio elegido es el de los hospitales rurales de baja complejidad, actualmente llamados hospitales comunitarios, que tienen importante presencia en la salud pública a nivel rural.

\section{Diseño de la investigación}

El diseño de esta investigación es no experimental ya que se realizará sin manipular las variables, observando los fenómenos en estudio tal y como se pueden dar en la vida cotidiana de los trabajadores en la organización, para después analizarlos. Además es de tipo transversal, ya que se realiza en un momento de tiempo determinado.

\section{Descripción del universo de estudio}

El universo de estudio de esta investigación corresponde a trabajadores distribuidos en cuatro hospitales comunitarios públicos. El 
criterio utilizado para describir la muestra es el de nivel de escolaridad, definido en tres niveles: Básica y Media, Técnico profesional, y Universitaria, ordenados de más a menos educación formal, niveles que influyen directamente en el acceso a cargos de mayor responsabilidad y autonomía en el área de la salud. La población total sobre la cual se aplicó el estudio corresponde a 400 individuos; se alcanzó una tasa de respuesta de $42 \%$, 172 funcionarios.

\begin{tabular}{|c|c|c|c|c|c|c|c|c|c|}
\hline \multicolumn{7}{|c|}{ TABLA 1: } \\
\hline \multicolumn{7}{|c|}{ Femenino } \\
\hline $\begin{array}{c}\text { Másica y } \\
\text { media }\end{array}$ & $\begin{array}{c}\text { Técnico } \\
\text { profesio- } \\
\text { nal }\end{array}$ & $\begin{array}{c}\text { Univer- } \\
\text { sitaria }\end{array}$ & Total & $\begin{array}{c}\text { Básica y } \\
\text { media }\end{array}$ & $\begin{array}{c}\text { Técnico } \\
\text { profesio- } \\
\text { nal }\end{array}$ & $\begin{array}{c}\text { Univer- } \\
\text { sitaria }\end{array}$ & Total & TOTAL \\
\hline $\begin{array}{c}\text { Cantidad } \\
(47 \%)\end{array}$ & $\begin{array}{c}36 \\
(27 \%)\end{array}$ & $\begin{array}{c}38 \\
(29 \%)\end{array}$ & $\begin{array}{c}\mathbf{1 3 1} \\
\mathbf{( 7 6 \% )}\end{array}$ & $\begin{array}{c}10 \\
(24,5 \%)\end{array}$ & $\begin{array}{c}10 \\
(24,5 \%)\end{array}$ & $\begin{array}{c}21 \\
(51 \%)\end{array}$ & $\mathbf{4 1}$ & $\begin{array}{c}\mathbf{( 2 4 \%}) \\
(100 \%)\end{array}$ \\
\hline $\begin{array}{c}\text { Promedio } \\
\text { edad }\end{array}$ & 47,66 & 38,38 & 37,26 & $\mathbf{4 1 , 6 4}$ & 42,20 & 42,70 & 34,38 & $\mathbf{3 7 , 3 6}$ & 40,26 \\
\hline $\begin{array}{c}\text { Desviación } \\
\text { estándar } \\
\text { edad }\end{array}$ & 9,81 & 10,47 & 10,30 & 11,71 & 11,75 & 13,66 & 8,66 & 10,41 & 12,18 \\
\hline
\end{tabular}

En la tabla 1 se observa que los trabajadores son $24 \%$ hombres y $76 \%$ mujeres, con una edad promedio de 37 y 41 años respectivamente.

Los niveles de escolaridad están divididos en tres: básica y media, que corresponde a los trabajadores que han terminado sus estudios entre la enseñanza básica y la licencia media; técnico-profesional, que corresponde a carreras técnicas o de pregrado con menos de cuatro ańos de duración; y universitaria, que corresponde a carreras de pregrado con cuatro ańos de duración o más. De la muestra femenina, un $44 \%$ pertenece al nivel de 
escolaridad de básica y media; un $27 \%$ al técnico profesional y un $29 \%$ al nivel universitario. En la muestra masculina, 24,5\% tiene nivel de básica y media, un $24,5 \%$ tiene nivel técnico profesional y un $51 \%$ universitario.

\section{Método de medición}

El instrumento utilizado en el presente estudio corresponde al Cuestionario para la evaluación del síndrome de quemarse por el trabajo CESQT de Gil-Monte (2007):

\begin{tabular}{|c|c|c|c|}
\hline \multicolumn{3}{|c|}{ TABLA 2: } \\
DISTRIBUCIÓN DE LAS PREGUNTAS DEL INSTRUMENTO DE MEDIDA \\
\hline Parte & Número Preguntas & Información Rescatada & \multicolumn{2}{c|}{ Tipo de respuesta } \\
\hline I. & 3 & $\begin{array}{c}\text { Información General del } \\
\text { encuestado. }\end{array}$ & Descriptiva \\
\hline II. & 20 & $\begin{array}{c}\text { Cuestionario CESQT } \\
\text { para medir Burnout }\end{array}$ & $\begin{array}{c}\text { 5, Nunca hasta Muy } \\
\text { frecuente, todos los } \\
\text { días. }\end{array}$ \\
\hline
\end{tabular}

* La utilización del instrumento CESQT para medir el Burnout fue autorizado por Víctor Olivares Faúndez, Professor of Organizational Psychology.

Este instrumento (descrito en las tablas 2 y 3 ) está formado por ítems que se valoran con una escala de frecuencia para que los individuos indiquen en qué grado experimentan cada una de las situaciones descritas en los elementos de la escala. Se aplica un rango de 5 adjetivos que van de "Nunca" (0) a "Todos los días" (4). 


\section{TABLA 3: DESCRIPCIÓN DE LAS ESCALAS DEL CUESTIONARIO CESQT}

\begin{tabular}{|c|l|l|}
\hline 1 & $\begin{array}{l}\text { Ilusión por el } \\
\text { Trabajo } \\
\text { (Invertido) }\end{array}$ & $\begin{array}{l}\text { Cinco ítems. Se define como el deseo del individuo de } \\
\text { alcanzar las metas laborales porque supone una fuente de } \\
\text { placer personal. Debido a que los ítems que componen } \\
\text { esta dimensión están formulados de manera positiva, ba- } \\
\text { jas puntuaciones en esta dimensión indican altos niveles } \\
\text { en SQT. }\end{array}$ \\
\hline $\mathbf{2}$ & Desgaste Psíquico & $\begin{array}{l}\text { Cuatro ítems. Se define como la aparición de agotamien- } \\
\text { to emocional y físico debido a que en el trabajo se tiene } \\
\text { que tratar a diario con personas que presentan o causan } \\
\text { problemas. }\end{array}$ \\
\hline $\mathbf{3}$ & Indolencia & $\begin{array}{l}\text { Seis ítems. Se define como la presencia de actitudes ne- } \\
\text { gativas de indiferencia y cinismo hacia los clientes de la } \\
\text { organización. Los individuos que puntúan alto en esta } \\
\text { dimensión muestran insensibilidad y no se conmueven } \\
\text { ante los problemas de los clientes. }\end{array}$ \\
\hline Culpa & $\begin{array}{l}\text { Cinco ítems. Se define como la aparición del sentimien- } \\
\text { to de culpa por el comportamiento y las actitudes ne- } \\
\text { gativas desarrolladas en el trabajo, en especial hacia las } \\
\text { personas con las que se establecen relaciones laborales. }\end{array}$ \\
\hline
\end{tabular}

\begin{tabular}{|c|c|c|c|c|c|}
\hline \multicolumn{6}{|c|}{ TABLA 4: } \\
PROPIEDADES PSICOMÉTRICAS DEL INSTRUMENTO DE BURNOUT \\
ANÁLISIS DE FIABILIDAD (VALORES 2008 Y ACTUALES)
\end{tabular}


Las estimaciones de la consistencia interna de las escalas se calcularon utilizando el coeficiente alfa de Cronbach (coeficiente de consistencia interna o indicador de unidimensionalidad). Gil-Monte (2008) demostró que CESQT es un instrumento que presenta validez factorial, y sus escalas presentan consistencia interna y validez concurrente, por lo que es un instrumento adecuado para evaluar el SQT en muestras de profesionales de enfermería (observar en la tabla 4, tercera columna); la fiabilidad de las cuatro escalas del instrumento original es alta y similar. Los coeficientes Alfa obtenidos en este estudio son variables y en todos los casos es adecuada, para la escala Indolencia el Alfa puede subir si se elimina el ítem 7. Sin embargo se decide dejar la escala completa porque el alfa es suficiente.

\section{Estrategia de recolección de datos}

Para lograr los objetivos descritos se procedió a la aplicación de un instrumento para la recolección de datos. El cuestionario fue entregado personalmente a los funcionarios, la participación de ellos fue de manera voluntaria y anónima.

La aplicación del cuestionario fue personal, autoaplicada y sin control de tiempo, esto permite ser contestada en forma autónoma por el encuestado, evita que se produzcan sesgos en los datos derivados del encuestador y encuestado, es decir, elimina posibles influencias que pudieran inducir una respuesta determinada.

\section{Resultados}

\section{Análisis Descriptivo}

Para el cálculo del resultado, es decir, conocer si cada sujeto presenta o no SQT, cada escala debe promediarse por separado. Estas cuatro columnas promedios deberán condicionarse respecto del valor 2, es decir, mayor o igual a 2 será $1 \mathrm{y}$, menor de 2 será 0 .

Para determinar la prevalencia del burnout se debe sumar los ítems de Ilusión, Indolencia y Desgaste, es decir, 15 ítems. Luego, igual que con las 4 escalas de burnout condicionar este resultado respecto de 1 y 0 . 


\begin{tabular}{|l|c|c|}
\hline \multicolumn{3}{|c|}{ TABLA 5: RESULTADOS POSIBLES DEL CESQT } \\
\hline Se puede obtener tres resultados distintos & Culpa & Prevalencia \\
\hline No presenta SQT & 0 & 0 \\
\hline $\begin{array}{l}\text { Perfil 1: Presenta trastorno pero no está } \\
\text { inhabilitado clínicamente }\end{array}$ & 1 & 0 \\
\hline $\begin{array}{l}\text { Perfil 2: Inhabilitado para trabajar sin } \\
\text { tratamiento clínico }\end{array}$ & 1 & 1 \\
\hline
\end{tabular}

Se entiende como "inhabilitado clínicamente" a la persona que presenta SQT y necesita tratamiento clínico para poder seguir trabajando, y puede ser considerado caso muy grave (Gil-Monte, 2005).

\begin{tabular}{|c|c|c|c|c|c|c|c|c|c|}
\hline & \multicolumn{4}{|c|}{ Femenino } & \multicolumn{4}{|c|}{ Masculino } & \multirow[b]{2}{*}{$\begin{array}{c}\text { TOTAL } \\
\text { Total }\end{array}$} \\
\hline & $\begin{array}{l}\text { Básica } \\
\text { y me- } \\
\text { dia }\end{array}$ & $\begin{array}{l}\text { Técnico } \\
\text { profe- } \\
\text { sional }\end{array}$ & $\begin{array}{c}\text { Univer- } \\
\text { sitaria }\end{array}$ & Total & $\begin{array}{c}\text { Básica } \\
\text { y me- } \\
\text { dia }\end{array}$ & $\begin{array}{c}\text { Técnico } \\
\text { profe- } \\
\text { sional }\end{array}$ & $\begin{array}{l}\text { Univer- } \\
\text { sitaria }\end{array}$ & Total & \\
\hline $\begin{array}{l}\text { No presen- } \\
\text { ta SQT }\end{array}$ & $\begin{array}{c}39 \\
(68 \%)\end{array}$ & $\begin{array}{c}28 \\
(78 \%)\end{array}$ & $\begin{array}{c}32 \\
(84 \%)\end{array}$ & $\begin{array}{c}99 \\
(76 \%)\end{array}$ & $\begin{array}{c}7 \\
(70 \%)\end{array}$ & $\begin{array}{c}7 \\
(70 \%)\end{array}$ & $\begin{array}{c}17 \\
(81 \%)\end{array}$ & $\begin{array}{c}31 \\
(76 \%)\end{array}$ & $\begin{array}{c}130 \\
(76 \%)\end{array}$ \\
\hline $\begin{array}{l}\text { Perfil 1: } \\
\text { Presenta } \\
\text { trastorno } \\
\text { pero no } \\
\text { está inha- } \\
\text { bilitado } \\
\text { clínica- } \\
\text { mente }\end{array}$ & $\begin{array}{c}12 \\
(21 \%)\end{array}$ & $\begin{array}{c}7 \\
(19 \%)\end{array}$ & $\begin{array}{c}5 \\
(13 \%)\end{array}$ & $\begin{array}{c}24 \\
(18 \%)\end{array}$ & $\begin{array}{c}1 \\
(10 \%)\end{array}$ & $\begin{array}{c}3 \\
(30 \%)\end{array}$ & $\begin{array}{c}3 \\
(14 \%)\end{array}$ & $\begin{array}{c}7 \\
(17 \%)\end{array}$ & $\begin{array}{c}31 \\
(18 \%)\end{array}$ \\
\hline $\begin{array}{l}\text { Perfil 2: } \\
\text { Inhabili- } \\
\text { tado para } \\
\text { trabajar sin } \\
\text { tratamien- } \\
\text { to clínico }\end{array}$ & $\begin{array}{c}6 \\
(11 \%)\end{array}$ & $\begin{array}{c}1 \\
(3 \%)\end{array}$ & $\begin{array}{c}1 \\
(3 \%)\end{array}$ & $\begin{array}{c}8 \\
(6 \%)\end{array}$ & $\begin{array}{c}2 \\
(20 \%)\end{array}$ & $\begin{array}{c}0 \\
(0 \%)\end{array}$ & $\begin{array}{c}1 \\
(5 \%)\end{array}$ & $\begin{array}{c}3 \\
(7 \%)\end{array}$ & $\begin{array}{c}11 \\
(6 \%)\end{array}$ \\
\hline TOTAL & $\begin{array}{c}57 \\
(100 \%)\end{array}$ & $\begin{array}{c}36 \\
(100 \%)\end{array}$ & $\begin{array}{c}38 \\
(100 \%)\end{array}$ & $\begin{array}{c}131 \\
(100 \%)\end{array}$ & $\begin{array}{c}10 \\
(100 \%)\end{array}$ & $\begin{array}{c}10 \\
(100 \%)\end{array}$ & $\begin{array}{c}25 \\
(100 \%)\end{array}$ & $\begin{array}{c}41 \\
(100 \%)\end{array}$ & $\begin{array}{c}172 \\
(100 \%)\end{array}$ \\
\hline
\end{tabular}


En la tabla 6, los resultados muestran respecto al segmento femenino, en el nivel de educación básica y media, que un $68 \%$ no presenta burnout, un $21 \%$ presenta trastorno pero no está inhabilitado clínicamente y un $11 \%$ está inhabilitado para trabajar sin tratamiento clínico. En el nivel de educación técnico-profesional, un $78 \%$ no presenta burnout, un 19\% presenta trastorno pero no está inhabilitado clínicamente y un 3\% está inhabilitado para trabajar sin tratamiento clínico. En el nivel de educación universitario, un $84 \%$ no presenta burnout, un $13 \%$ presenta trastorno pero no está inhabilitado clínicamente y un 3\% está inhabilitado para trabajar sin tratamiento clínico.

Respecto al segmento masculino, los resultados muestran, en el nivel de educación básica y media, que un $70 \%$ no presenta burnout, un $10 \%$ presenta trastorno pero no está inhabilitado clínicamente y un 20\% está inhabilitado para trabajar sin tratamiento clínico. En el nivel de educación técnicoprofesional un $70 \%$ no presenta burnout y un 30\% presenta trastorno pero no está inhabilitado clínicamente. No hay casos que estén inhabilitados para trabajar sin tratamiento clínico. En el nivel de educación universitario un 81\% no presenta burnout, un $14 \%$ presenta trastorno pero no está inhabilitado clínicamente y un 5\% está inhabilitado para trabajar sin tratamiento clínico.

Si unimos las muestras femenina y masculina, los resultados a nivel general muestran que un $76 \%$ de los funcionarios no presenta burnout, un $18 \%$ presenta trastorno pero no está inhabilitado clínicamente y un $6 \%$ está inhabilitado para trabajar sin tratamiento clínico.

\section{Análisis de correlaciones}

TABLA 7: CORRELACIONES DE BURNOUT CON EDAD Y NIVEL DE ESCOLARIDAD

\begin{tabular}{|c|c|c|}
\hline & Edad & Nivel de escolaridad \\
\hline Burnout Femenino &, 038 &,$- 176^{*}$ \\
& $(0,661)$ & $(0,044)$ \\
\hline Burnout Masculino &, 218 &,- 170 \\
& $(0,172)$ & $(0,287)$ \\
\hline
\end{tabular}

* La correlación es significativa al nivel 0,05 (bilateral).

** La correlación es significativa al nivel 0,01 (bilateral).

Los valores entre paréntesis son los valores $p$. 
En la tabla 7, los resultados muestran que la variable "Burnout Femenino" posee una correlación estadísticamente significativa, baja y negativa con el "nivel de escolaridad". Esto quiere decir que a medida que las funcionarias tengan un nivel de escolaridad mayor tenderá a disminuir el burnout en ellas. La muestra masculina no presenta correlación estadísticamente significativa entre burnout y nivel de escolaridad.

\section{Conclusiones y discusión}

El burnout o síndrome de estar quemado por el trabajo (SQT) constituye uno de los daños laborales de carácter psicosocial más importantes en la sociedad actual. El estudio del burnout no es una cuestión de modas y de unos cuantos, sino una necesidad social orientada a mejorar la salud y la calidad de vida de las personas.

En la Nota Técnica de Prevención sobre burnout se muestran los principales factores psicosociales responsables del desarrollo del síndrome.

Respecto a las variables individuales se destaca el género (siendo las mujeres las que puntúan generalmente más alto en agotamiento e ineficacia profesional) y variables de personalidad (son las personas con un patrón de conducta Tipo A, baja estabilidad emocional y locus de control externo las que son más vulnerables de sufrir burnout). A estas variables individuales, se añade también la falta de eficacia profesional como antecedente proximal del burnout. A nivel social se destaca el papel de la percepción de falta de apoyo social que proviene de las redes sociales del individuo. A nivel organizacional se destacan los aspectos relacionados con el contenido del puesto (puestos "tóxicos"), la falta de un clima laboral adecuado o la falta de reciprocidad (Bresó, Salanova, Schaufeli \& Nogareda, 2007b).

Los datos para este análisis fueron obtenidos a través del cuestionario CESQT, instrumento para la evaluación del SQT, desarrollado por Gil-Monte, (2005) y que permite obtener tres resultados posibles; No presenta SQT, Perfil 1 (Presenta trastorno pero no está inhabilitado clínicamente) y Perfil 2 (Inhabilitado para trabajar sin tratamiento clínico). 
Como se indicó en la parte teórica, Gil-Monte (2005) establece la existencia de dos perfiles de individuos en el desarrollo del SQT. Por una parte, individuos (Tipo 1) a los que las estrategias de afrontamiento cognitivas que permiten justificar la utilización de conductas proscritas por la ética les resultan de utilidad. Por el contrario, el segundo perfil (Tipo 2) son individuos que experimentan remordimientos por no cumplir de manera adecuada las prescripciones del rol, por sentirse desgastados y no poder dar más de sí mismos, y por la utilización de estrategias de afrontamiento que conllevan un trato negativo e impersonal con los clientes. Probablemente, esos individuos experimentarán con frecuencia sentimientos de culpa hasta el punto de necesitar la desvinculación, acudirán con más frecuencia al médico, y manifestarán con mayor frecuencia e intensidad problemas psicosomáticos vinculados al estrés laboral.

Gil-Monte (2008) demostró que CESQT es un instrumento adecuado para evaluar el SQT en muestras de profesionales de enfermería. La actual muestra es del área de servicio, específicamente del área de la salud, incluye todo el personal de un hospital público. Los resultados obtenidos prueban la hipótesis formulada por Gil-Monte (2008) de que los futuros estudios llevarán a conclusiones similares con otros colectivos ocupacionales de servicio.

Los resultados obtenidos muestran una mayor presencia del Perfil 2 en la muestra con el nivel de escolaridad más bajo (básico y medio), esto tanto para la muestra femenina como masculina. También se observa que el Perfil 1, es más alto en la muestra femenina con el nivel de escolaridad más bajo (básico y medio) y en la muestra masculina con el nivel de escolaridad técnico profesional. Esto permite concluir que el nivel de escolaridad influye en la aparición del burnout para las personas del área de la salud; específicamente el segmento auxiliar de Servicio, Administrativo y Técnico paramédico estaría más expuesto a sufrir de burnout. Por lo tanto se podría esperar que personas con un nivel de escolaridad universitaria pudieran tener mayores recursos para enfrentar el burnout. Por ejemplo, formación superior (universitaria) le da a la trabajadora mayor sentido de eficacia, permitiéndole mayor control sobre su trabajo y mayor autonomía. Este resultado está basado en una correlación estadísticamente significativa 
baja, por lo que no es altamente concluyente (se observa una tendencia), sin embargo los resultados presentados en la tabla descriptiva lo confirman y la Nota técnica de Bresó et al. (2007b), coincide al indicar que las mujeres puntúan generalmente más alto en agotamiento e ineficacia profesional. Queda una duda respecto a la correlación del burnout masculino con el nivel de escolaridad que no es estadísticamente significativa, sin embargo los resultados descriptivos indican algo similar a lo que indican con el género femenino, donde el mayor porcentaje de burnout considerado "caso grave"(Perfil 2) se observa en las personas con un nivel de escolaridad básica y media.

En una revisión sobre burnout realizada por Schaufeli (2003) se muestran datos sobre la prevalencia del burnout en muestras holandesas: entre un $4 \%$ y un $7 \%$ de los trabajadores podían considerarse población de riesgo (llegando al 10\% en algunas ocupaciones específicas), mientras que un 7,2\% sufrían burnout clínico, lo que implica entre 250.000 y 440.000 afectados. Esta situación puede haberse agravado en España debido a los incrementos del estrés que pueden observarse en las encuestas españolas sobre condiciones de trabajo (48\%) (5ª Encuesta Nacional sobre Condiciones de Trabajo, 2004).

Si se considera a las personas con el Perfil 1 como población de riesgo, el porcentaje obtenido en este estudio es de $18 \%$ y a las personas con el Perfil 2 como burnout clínico es de 6\%. Comparando estos resultados con la muestra holandesa de Schaufeli (2003) se observa que en la muestra chilena hay un mayor porcentaje de población de riesgo y en burnout clínico en la muestra chilena hay un menor porcentaje.

Así, se puede concluir que en la gestión clínica hospitalaria, debido a que las personas están expuestas a situaciones de presión y tensión en forma frecuente y que atienden a personas que presentan necesidades inclusive vitales, es conveniente mantener un sistema de vigilancia epidemiológica en torno al Síndrome de Quemarse en el Trabajo, con el fin de prevenir su aparición en las entidades de salud, sobre todo en los trabajadores con niveles de baja escolaridad, que parecen ser los más sensibles a la presión ya que no tienen forma de controlarla.

Por otra parte, se hace necesario contar con equipos de salud en los hospitales que atiendan a los trabajadores de los establecimientos que 
presenten depresión, duelos prolongados, o trastornos ansiosos. También es conveniente estimular el trabajo en equipo en los establecimientos debido a que es un factor protector frente a la aparición del burnout. Desde el punto de vista de las acciones de un bienestar en la institución es conveniente que se propongan actividades que beneficien el equilibrio entre trabajo y familia.

Cuando el resultado de la evaluación ha detectado una falta de recursos personales, debieran utilizarse estrategias centradas en el individuo con el objetivo de aumentar y mejorar sus recursos personales. Estas estrategias suponen la implicación activa del trabajador ya que pretenden dotarle de conocimientos, generar competencias, desarrollar habilidades, etc.

Cuando el problema está en que existen altas demandas laborales y/o bajos recursos laborales, el foco no está tanto en el individuo sino en la propia organización, y por tanto, las estrategias van orientadas a mejorar la situación laboral, como por ejemplo aumentando la variedad de tareas, o resolviendo conflictos de rol, escogiendo el estilo de liderazgo adecuado a la situación y a los seguidores, etc.

\section{Referencias}

Baumeister, R.F., Stillwell, A. M. \& Heatherton, T.F. (1994). Guilt: An interpersonal approach. Psychological Bulletin 115, 243-267.

Bresó, E., Salanova, M. \& Schaufeli, W.B. (2007a). In search of the 'third dimension' of burnout. Applied Psychology: An International Review, 56, 460-478.

Bresó, E., Salanova, M., Schaufeli, W. B. \& Nogareda, C. (2007b). Síndrome de estar quemado por el trabajo "Burnout" (III): Instrumento de medición. Nota Técnica de Prevención, 732, $21^{a}$ Serie. Instituto Nacional de Seguridad e Higiene en el trabajo.

Cherniss, C. (1993). The role of professional self-efficacy in the etiology and amelioration of burnout. En W. B. Schaufeli, C. Maslach \& T. Marek (Eds.), Professional burnout: Recent development in theory and research (pp. 135-149). Washington, DC: Taylor \& Francis.

Farber, B.A., \& Miller, J. (1981). Teacher burnout: A psycho-educational perspective. Teacher College Record, 83(2), 235-243. 
Freudenberger, H. J. (1974). Staff burnout. Journal of Social Issues, 30, 159-165. Green, D. E., Walkey, F. H. \& Taylor, A. J. W. (1991). The three-factor structure of the Maslach Burnout Inventory. Journal of Science Behavior and Personality, 6, 453-472.

Gil-Monte, P. R. (2005). El sindrome de quemarse por el trabajo: una enfermedad laboral en la sociedad del bienestar. Madrid: Pirámide.

Gil-Monte, P. R. (2007). El síndrome de quemarse por el trabajo (Burnout): una perspectiva histórica. En P. R. Gil-Monte \& B. Moreno-Jiménez (Comps.), El sindrome de quemarse por el trabajo (burnout): grupos profesionales de riesgo (en prensa). Madrid: Pirámide.

Gil-Monte, P. R. (2008). Evaluación psicométrica del síndrome de burnout: el cuestionario "CESQT". En J. Garrido (Comp.), „Maldito trabajo! (pp. 269-291). Barcelona: Granica.

Llorens, S., García, M. \& Salanova, M. (2005). Burnout como consecuencia de una crisis de eficacia: un estudio longitudinal en profesores de secundaria. Revista de Psicología del Trabajo y de las Organizaciones, 21, 55-70.

Maslach, C. (1976). Burned-out. Human Behaviour, 5, 16-22.

Maslach, C. (1982). Burnout: A social psychological analysis. En J.W. Jones (Comp.), The burnout syndrome: Current research, theory, interventions (pp.30-53). Park Ridge, Illinois: London House Press.

Maslach, C. (1993). Burnout: A multidimensional perspective. En W. B. Schaufeli, C. Maslach \& T. Marek (Eds.), Professional burnout (pp. 19-32). Washington, DC: Taylor and Francis.

Maslach, C. (2009). Comprendiendo el burnout. Revista Ciencia \& Trabajo, 32, 37-43.

Maslach, C., Schaufeli, W. B. \& Leiter, M. P. (2001). Burnout. Annual Review of Psychology, 52, 397-422.

Ministerio de Trabajo y Asuntos Sociales. Instituto Nacional de Seguridad e Higiene en el Trabajo España (2004). 5a Encuesta Nacional sobre Condiciones de Trabajo.

Paine, W.S. (1982). The burnout syndrome in context. En J.W. Jones (Comp.), The burnout syndrome: Current research, theory, interventions (pp.1-29). Park Ridge, Illinois: London House Press.

Pines, A. (1983). On burnout and buffering effects of social support. En B.A. Farber (Comp.), Stress and burnout in the human services professions (pp.155-174). Nueva York: Pergamon Press.

Price, D. M., \& Murphy, P.A (1984): Staff burnout in the perspective of grief theory. Death Education, 8(1), 47-58.

Salanova, M. (2006). Medida y evaluación del burnout: nuevas perspectivas. En P.R.Gil-Monte, M. Salanova, J.L. Aragón \& W. Schaufeli, 
(Eds). El sindrome de quemarse por el trabajo en Servicios Sociales (pp. 27-43). Valencia: Diputación de Valencia.

Salanova, M., Martínez, I., Bresó, E., Llorens, S. \& Grau, R. (2005). Bienestar psicológico en estudiantes universitarios: facilitadores y obstaculizadores del desempeño académico. Anales de Psicología, 21, 170-180.

Salanova, M., Schaufeli, W. B., Llorens, S., Peiró, J. M., \& Grau, R. (2000). Desde el "burnout" al "engagement": ¿Una nueva perspectiva? Revista de Psicología del Trabajo y de las Organizaciones, 16, 117-134.

Schaufeli, W.B. (2003). Past performance and future perspectives of burnout research. Journal of Industrial Psychology, 29, 1-15.

Schaufeli, W.B. \& Buunk, B.P. (2002). Burnout: An overview of 25 years of research and theorizing. En M. J. Schabracq, J. A. M. Winnubst \& C. L. Cooper (Eds.), The Handbook of Work and Health Psychology (2nd Edition, pp. 383-425). Chichester: John Wiley \& Sons.

Schaufeli, W.B. \& Enzmann, D. (1998). The burnout companion to study and practice: a critical analysis. London: Taylor \& Francis.

Schaufeli, W.B. \& Salanova, M. (2007). Efficacy or inefficacy, that's the question: Burnout and engagement, and their relationships with efficacy beliefs. Anxiety, Coping \& Stress 20, 177-196.

Fecha de recepción: 16 de agosto de 2010.

Fecha de aceptación: 07 de junio de 2011. 